\title{
Disseminated Tumor Cells in Bone Marrow of Gastric Cancer Patients: Correlation with Tumor Hypoxia and Clinical Relevance
}

\author{
Larissa Bubnovskaya, ${ }^{1}$ Antonina Kovelskaya, ${ }^{1}$ Lilya Gumenyuk, \\ Irina Ganusevich, ${ }^{1}$ Lesya Mamontova, ${ }^{1}$ Victor Mikhailenko, ${ }^{1}$ Dmitry Osinsky, \\ Sergej Merentsev, ${ }^{2}$ and Sergej Osinsky ${ }^{1}$ \\ ${ }^{1}$ R.E. Kavetsky Institute of Experimental Pathology, Oncology and Radiobiology, National Academy of Sciences of Ukraine, \\ Vasilkovskaya Street 45, Kiev 03022, Ukraine \\ ${ }^{2}$ City Clinical Oncological Center, Verchovynna Street 69, Kiev 03115, Ukraine
}

Correspondence should be addressed to Sergej Osinsky; osinskysp12@ukr.net

Received 12 September 2013; Revised 21 December 2013; Accepted 2 January 2014; Published 11 February 2014

Academic Editor: Paul Magnus Schneider

Copyright (C) 2014 Larissa Bubnovskaya et al. This is an open access article distributed under the Creative Commons Attribution License, which permits unrestricted use, distribution, and reproduction in any medium, provided the original work is properly cited.

\begin{abstract}
Aim. The evaluation of the clinical relevance of disseminated tumor cells (DTCs) in bone marrow (BM) of patients with gastric cancer (GC) and their association with primary tumor hypoxia. Patients and Methods. 89 resected specimens were used. DTCs were detected using immunocytochemistry, the level of tumor hypoxia using NMR spectroscopy, CD68, CD34, VEGF, and VEGFR-1 (Flt1) expression using immunohistochemistry, and MMP-2 and MMP-9 activity using zymography. Results. DTCs were detected in $51.4 \%$ of GC patients with $\mathrm{M}_{0}$. There was significant correlation between frequency of DTCs in BM and level of tumor hypoxia $(P<$ 0.024). DTCs presence was accompanied with Flt-1 positivity of BM. The correlation between DTCs and tumor VEGF expression in patients with $\mathrm{M}_{0}$ was shown $(P<0.0248)$. Activity of MMP-2 and MMP-9 in BM was linked with DTCs in patients with $\mathrm{M}_{0}$ $(P<0.05)$. Overall survival (OS) of patients with $\mathrm{M}_{0}$ and DTCs was shorter than that of patients without DTCs (patients in both groups were operated only) $(P=0.0497)$. Conclusion. Appearance of DTCs correlates with hypoxia level in primary tumors. Detection of DTCs in GC patients may be relevant indicator for adjuvant chemotherapy using.
\end{abstract}

\section{Introduction}

Gastric cancer is one of the most common cancers in Europe ranking the fifth after lung, prostate, colorectal, and bladder cancers in men and breast, colorectal, lung, and cancer of the corpus uteri in women [1]. In Ukraine in 2011 the annual age-standardized incidence rate was 21.5/100.000 ranking the fourth after lung, skin, and prostate cancers in men and 8.8/100 000 ranking the seventh after breast, skin, corpus uteri, colon, cervix uteri, and rectal cancers in women [2]. The therapy outcome of gastric cancer is still not satisfactory, and distant metastasis is kept as a key factor in the unfavorable results of gastric cancer treatment. It is known that even curative resection can not always guaranty expected longterm results of therapy that may be explained by the early dissemination of tumor cells even still before the surgical intervention [3-5]. It is shown that "tumor cells can disseminate from the earliest preneoplastic lesions, sometimes even before the formation of overt primary tumors" [6]. At the same time it is unable to detect early metastasis up till now despite modern tools, for example, MRT, PET.

Tumor cells leaving primary site can mainly settle in bone marrow (BM) with potency to form the metastases. These cells named disseminated tumor cells (DTCs) cannot be detected by conventional cytological methods, but they may be found both by immunocytochemistry and molecular technologies.

It was shown that DTCs in BM may be detected in 25$60 \%$ of patients with different tumors categorized as $\mathrm{M}_{0}[4-$ 7]. Conception of the detection of micrometastases in BM 
has been introduced in clinical practice more than 30 years ago [8]. BM is considered now as a common homing-organ for DTCs that escape from epithelial tumor, first of all from breast, lung, prostate, and colorectal cancers. It was shown that persistent DTCs in BM are associated with unfavorable prognosis for breast cancer patients [9].

Perhaps firstly publication concerning DTCs in gastric cancer patients appeared in 1991 when Schlimok et al. [10] have shown the presence of DTCs in BM of 30\% patients with gastric cancer without obvious distant metastases. The correlation between DTCs and some clinicopathological characteristics, in particular regional and distant metastasis, and Lauren classification was also determined. Further studies confirmed these data and focused one's attention on methodology of DTCs search and pointed out the clinical relevance of DTCs in gastric cancer patients, in particular their negative impact on survival [11-17]. Kolodziejczyk et al. [18] studying the influence of neoadjuvant chemotherapy on DTCs in gastric cancer patients have shown that frequency of DTCs finding was significantly decreased after chemotherapy but without substantial impact on disease outcome. Nevertheless, authors proposed to continue these studies, in particular in multicenter protocol investigations. At the same time some observations have been published where the clinical relevance of DTCs in gastric cancer patients was not observed [19-21]. Recently published review of Bidard et al. [22] allows to conclude that problem of DTCs in gastric cancer is relevant for clinics but it is not fully solved and needs to be clarified to improve the treatment outcome.

Our study was aimed to evaluate the influence of DTCs in BM of gastric cancer patients on survival and their prognostic significance. Moreover, our attention was focused on the assessment of possible correlation between DTCs in $\mathrm{BM}$ and hypoxia profile in primary tumor exploiting wellknown fact that solid tumors are hypoxic that mediates tumor aggressiveness and poor disease outcome [23].

\section{Patients and Methods}

2.1. Patients. A total of 89 patients (62 men and 27 women) with primary gastric cancer (GC) were diagnosed and treated at the City Clinical Oncological Center (Kiev), during period 2008-2011 (Table 1). No patient received chemotherapy or radiation prior to surgery. Tissue samples were taken immediately after tumor excision. Tumors were classified and staged according to the 2002 version of the UICC staging system [24]. Histological types of tumor were evaluated by WHO histological classification (2000) [25]. All patients were thoroughly informed about the study that was approved by the local ethics committee.

2.2. Detection of Tumor Cells in Bone Marrow. Preoperatively, 2.0-3.0 mL of BM aspirates from the sternum with conventional cautions to avoid the hit of skin epithelial cells into the sample was taken into a heparinized syringe and transferred into a tube "Sarstedt" containing EDTA K. After Ficoll-Hypaque density centrifugation (density, 1.077; Sigma-Aldrich, USA) to isolate the mononuclear cell fraction (1105 g for 20 minutes), the interphase was washed twice in
TABLE 1: Patient and tumor characteristics.

\begin{tabular}{|c|c|}
\hline Characteristics & Number, $89(\%)$ \\
\hline \multicolumn{2}{|l|}{ Gender } \\
\hline Male & $62(69.7)$ \\
\hline Female & $27(30.3)$ \\
\hline \multicolumn{2}{|l|}{ Age (years) } \\
\hline Median & 62 \\
\hline Range & $34-84$ \\
\hline \multicolumn{2}{|l|}{ Tumor location } \\
\hline Upper third & $9(10.1)$ \\
\hline Middle third & $29(32.6)$ \\
\hline Lower third & $47(52.8)$ \\
\hline Total & $4(4.5)$ \\
\hline \multicolumn{2}{|l|}{ UICC stage } \\
\hline I & $22(24.7)$ \\
\hline II & $16(18.0)$ \\
\hline III & $23(25.8)$ \\
\hline IV & $28(31.5)$ \\
\hline \multicolumn{2}{|l|}{ Histological type } \\
\hline Adenocarcinoma & $61(68.5)$ \\
\hline Mucinous adenocarcinoma & $12(13.5)$ \\
\hline Signet-ring cell carcinoma & $13(14.6)$ \\
\hline Undifferentiated carcinoma & $3(3.4)$ \\
\hline \multicolumn{2}{|l|}{ Grade $(\mathrm{G})$} \\
\hline 1 & $6(6.7)$ \\
\hline 2 & $15(16.9)$ \\
\hline 3 & $60(67.4)$ \\
\hline 4 & $8(9.0)$ \\
\hline \multicolumn{2}{|l|}{ T-classification } \\
\hline $\mathrm{T}_{1}$ & $10(11.2)$ \\
\hline $\mathrm{T}_{2}$ & $18(20.2)$ \\
\hline $\mathrm{T}_{3}$ & $33(37.1)$ \\
\hline $\mathrm{T}_{4}$ & $28(31.5)$ \\
\hline \multicolumn{2}{|l|}{ Nodal involvement } \\
\hline $\mathrm{N}_{0}$ & $43(48.3)$ \\
\hline $\mathrm{N}_{1}$ & $17(19.1)$ \\
\hline $\mathrm{N}_{2}$ & $29(32.6)$ \\
\hline \multicolumn{2}{|l|}{ Distant metastasis } \\
\hline $\mathrm{M}_{0}$ & $70(78.6)$ \\
\hline $\mathrm{M}_{1}$ & $19(21.4)$ \\
\hline
\end{tabular}

phosphate-buffered saline (PBS) with removing of erythrocytes (Uti-Lyse Erythrocyte Lysing Reagent, Dako Cytomation, USA), resuspended to a concentration of $570 \cdot 10^{3}$ cells $/ 30 \mu \mathrm{L}$, and cytocentrifuged on glass slides. Specimens were air-dried from 12 to 24 hours and stained immediately or stored at $-20^{\circ} \mathrm{C}$.

Detection of tumor cells (cytokeratin-positive cells, CKpositive cells) in BM cytospin preparations fixed in acetone was provided by APAAP method (alkaline phosphataseantialkaline phosphatase) and visualization system EnVision G/2 System/AP Rabbit/Mouse (Permanent Red) (Dako Cytomaiton, Denmark). Monoclonal mouse antibodies against 
panCK (clone AE1/AE3, Dako Cytomation, Denmark) were used as primary antibodies. Each assay was controlled negatively by staining of one cytospin preparation with

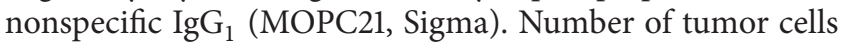
(CK-positive cells) was expressed on $10^{6} \mathrm{BM}$ mononuclear cells. BM samples were scored "positive" if the presence of two or more CK-positive cells per $10^{6}$ mononuclear cells was detected (from 6 to 12 slides per patient were screened).

\subsection{Detection of Flt-1-Positive Cells in Bone Marrow with} Immunohistochemical Method. Cytospins were fixed by formol-acetone solution ( $\mathrm{pH}$ 6.6) in accordance with the instruction. Slides were treated by $0.3 \%$ Triton X-100 solution, washed by PBS and blocking of endogenous peroxidase followed by incubation in $3 \%$ bovine serum albumin to switch off nonspecific reaction antigen-antibody. Cytospins were incubated with primary polyclonal rabbit antibodies against Flt-1 (sc-316, Santa Cruz Biotechnology, Inc., USA) in optimal dilution $1: 80$ within $1 \mathrm{~h}$. After washing of primary antibodies slides were processed with PolyVueHRP Detection System Components (Diagnostic BioSystems, USA).

\subsection{Immunohistochemical Examination of Tumor Tissue.} Expression of CD34 (the endothelial cell marker), CD68 (the commonly used macrophage marker), and VEGF was evaluated on deparaffinized slides by means of immunohistochemical staining using specific monoclonal mouse antibodies: clone QBEnd 10 (1:100), clone PG-M1 (1:80), and clone VG1 (1:50), respectively. Immunoreactions were detected and visualized with the polymer-peroxidase method (EnVision+/HRP and 3,3-diaminobenzidine; Dako Cytomation, Denmark) followed by counterstaining with Mayer hematoxylin. Positive controls were used as monoclonal antibodies against cytokeratins (clone MNF116, DakoCytomation, Denmark). Nonimmunized serum or PBS was substituted by primary antibodies as the negative control.

Microvessel density (MVD), detected by immunostaining for CD34, was assessed by the hot spot method [26]. CD68positive cells were counted per 1000 cells in each slide and the number of CD68-positive cells was reported as percent. VEGF expression was assessed by scoring the number of all positive cells per 200x field. When the tumor consisted of less than $25 \%$ immunoreactive cells, the case was scored as weak, cases with $26-50 \%$ immunoreactive cells were scored as moderate, and those with $51-100 \%$ immunoreactive cells were scored as strong.

Expression of VEGFR-1 (Flt-1) was detected using polyclonal rabbit antibodies against Flt-1 (sc-316 (1:80), Santa Cruz Biotechnology, Inc., USA). Normal rabbit IgG (Dako Cytomation, Denmark) was used for the negative control. When the tumor consisted of more than $10 \%$ immunoreactive cells, the case was scored as positive.

2.5. Metalloproteinase-2 and Metalloproteinase-9 Activity Assay. Tumor specimens and BM aspirates $(0.5 \mathrm{~mL})$ were placed into the liquid nitrogen up to processing. Activity of MMP-2 and MMP-9 was determined for each sample by zymography in $12 \%$ polyacrylamide gel with SDS and $0.1 \%$ of gelatin as substrate [27].

2.6. ${ }^{31} P$ NMR Spectroscopy. Level of tumor hypoxia was assessed with ${ }^{31} \mathrm{P}$ NMR spectroscopy. ${ }^{31} \mathrm{P}$ NMR spectra of perchloric acid (PCA) tumor extracts were acquired by means of a high-resolution Bruker $400 \mathrm{MHz}$ spectrometer (Widebore Ultrashield, AV-400 electronics, Germany) using a probe of $5 \mathrm{~mm}$ inner diameter. All details of method were presented in our earlier publication [27].

2.7. Statistical Analysis. All statistical analyses were conducted using the NCSS 2000/PASS 2000 and Prism, version 4.0 software packages. Correlations were analyzed with the Pearson correlation coefficient. The survival proportion was estimated by using the Kaplan-Meier method and differences in survival were analyzed with the log-rank test. Prognostic values of relevant variables were analyzed by means of the Cox proportional hazards model using hazard ratio and $\chi^{2}$ test. Two-tailed $P$ values $<0.05$ were considered statistically significant.

\section{Results}

3.1. Tumor Cells in Bone Marrow and Their Correlation with Clinical Variables. Individual patient data from a total of 89 histologically confirmed gastric cancer patients were included in this study (Table 1). The median age was 62 years. Overall, 51 patients (57.3\%) had DTCs during follow-up. The mean number of DTCs in BM was $5.5 \pm 1.0 / 10^{6}$ mononuclear cells. Patients with category $\mathrm{M}_{0}$ had DTCs in BM in $51.4 \%$ of cases $\left(6.5 \pm 2.4 \mathrm{CK}\right.$-positive cells per $10^{6}$ mononuclear cells), and patients with category $M_{1}$ in $78.9 \%$ cases $(8.0 \pm$ 2.0 CK-positive cells per $10^{6}$ mononuclear cells). There was no association of DTCs in BM with clinicopathological characteristics (Table 2). Meanwhile, the association between the presence of CK-positive cells in BM and level of hypoxia in primary tumor was detected: severe and moderate hypoxia was found in $75 \%$ of primary tumors in patients with DTCs in BM while mild and weak hypoxia in $32.2 \%$ only $(P<$ $0.01)$. Level of tumor hypoxia assessed by NMR spectroscopy [27] was ranged as follows: if the $\mathrm{PME} / \mathrm{Pi}<1.0$, tumors are characterized by severe hypoxia, $1.0<\mathrm{PME} / \mathrm{Pi}<1.4$ moderate hypoxia, $1.4<\mathrm{PME} / \mathrm{Pi}<2.0$ mild hypoxia, and $\mathrm{PME} / \mathrm{Pi}>2.0$ weak hypoxia (satisfactory oxygenation).

It was also determined that the probability of appearance of tumor cells in BM of patients with category $\mathrm{M}_{0}$ is increased by a factor of 11.4 (odds ratio $11.4,95 \%$ CI 2.71-47.89, $\chi^{2}=$ $12.3, P<0.001)$ when tumors were characterized by severe and moderate hypoxia.

3.2. Flt-1-Positive Cells in Bone Marrow and Primary Tumor. It was found that Flt-1 positive cells were detected both in BM and tumor, in $58.5 \%$ and $79 \%$ of patients, respectively. The presence of CK-positive cells in BM was accompanied with the Flt-1-positivity of BM in $67 \%$ and the absence of CK-positive cells with the Flt-1-positivity in $45 \%$ of cases. The correlation between the number of Flt-1-positive tumor and 
TABLE 2: Prevalence of disseminated tumor cells in bone marrow by clinical variables.

\begin{tabular}{|c|c|c|c|}
\hline Variables & All patients $(n=89,100 \%)$ & Patients with DTC $(n=51,57.3 \%)$ & Patients without DTC $(n=38,42.7 \%)$ \\
\hline \multicolumn{4}{|l|}{ Gender } \\
\hline Male & 62 & $27(43.5)$ & $35(56.5)$ \\
\hline Female & 27 & $17(63.0)$ & $10(37.0)$ \\
\hline \multicolumn{4}{|l|}{ Patients age groups $(n, \%)$} \\
\hline$\leq 65$ & 56 & $35(62.5)$ & $21(37.5)$ \\
\hline$>65$ & 33 & $16(48.5)$ & $17(51.5)$ \\
\hline Age (years; median, range) & $62(34-84)$ & & \\
\hline \multicolumn{4}{|l|}{ Tumor location } \\
\hline Upper third & 9 & $6(66.7)$ & $3(33.3)$ \\
\hline Middle third & 29 & $13(44.8)$ & $16(55.2)$ \\
\hline Lower third & 47 & $22(46.8)$ & $25(63.2)$ \\
\hline Total & 4 & $3(75.0)$ & $1(25.0)$ \\
\hline \multicolumn{4}{|l|}{ UICC Stage } \\
\hline I & 22 & $11(50.0)$ & $11(50.0)$ \\
\hline II & 16 & $6(37.5)$ & $10(62.5)$ \\
\hline III & 23 & $10(43.5)$ & $13(56.5)$ \\
\hline IV & 28 & $17(60.7)$ & $11(39.3)$ \\
\hline \multicolumn{4}{|l|}{ Histological type } \\
\hline Adenocarcinoma & 61 & $26(42.6)$ & $35(57.4)$ \\
\hline Mucinous adenocarcinoma & 12 & $8(66.7)$ & $4(33.3)$ \\
\hline Signet-ring carcinoma & 13 & $8(61.5)$ & $5(38.5)$ \\
\hline Undifferentiated carcinoma & 3 & $2(66.7)$ & $1(33.3)$ \\
\hline \multicolumn{4}{|l|}{ Grade $(\mathrm{G})$} \\
\hline 1 & 6 & $3(50.0)$ & $3(50.0)$ \\
\hline 2 & 15 & $4(26.7)$ & $11(73.3)$ \\
\hline 3 & 60 & $33(55.0)$ & $27(45.0)$ \\
\hline 4 & 8 & $4(50.0)$ & $4(50.0)$ \\
\hline \multicolumn{4}{|l|}{ T-classification } \\
\hline $\mathrm{T}_{1}$ & 10 & $3(30.0)$ & $7(70.0)$ \\
\hline $\mathrm{T}_{2}$ & 18 & $13(72.2)$ & $5(27.8)$ \\
\hline $\mathrm{T}_{3}$ & 33 & $15(45.6)$ & $18(54.5)$ \\
\hline $\mathrm{T}_{4}$ & 28 & $13(46.4)$ & $15(53.6)$ \\
\hline \multicolumn{4}{|l|}{ Nodal involvement } \\
\hline $\mathrm{N}_{0}$ & 43 & $18(41.9)$ & $25(58.1)$ \\
\hline $\mathrm{N}_{1}$ & 17 & $9(52.9)$ & $8(47.1)$ \\
\hline $\mathrm{N}_{2}$ & 29 & $17(58.6)$ & $12(41.4)$ \\
\hline \multicolumn{4}{|l|}{ Distant metastasis } \\
\hline $\mathrm{M}_{0}$ & 70 & $36(51.4)$ & $34(48.6)$ \\
\hline $\mathrm{M}_{1}$ & 19 & $15(78.9)$ & $4(21.1)$ \\
\hline \multicolumn{4}{|l|}{ Systemic therapy } \\
\hline Operation only & 47 & $27(57.4)$ & $20(42.6)$ \\
\hline Adjuvant chemotherapy & 42 & $24(57.1)$ & $18(42.9)$ \\
\hline
\end{tabular}

Flt-1-positive BM was not observed. The probability of the presence of Flt-1-positive cells in BM was increased by a factor of 2.7 when tumors were characterized by severe and moderate hypoxia although this probability was not statistically significant (odds ratio $=2.7 ; 95 \%$ CI $1.76-4.72 ; P>0.05$ ). The mean number of Flt-1-positive cells in tumor was $34 \pm 3.0 \%$ (median 47\%, range 0-96).
3.3. VEGF-Positive Cells, CD68, and MVD in Primary Tumor and CK-Positive Cells in Bone Marrow. Positive reaction with monoclonal antibody to VEGF was observed in $73 \%$ of tumors. It was shown that CK-positive cells in BM were detected in $73 \%$ of patients with VEGF-positive tumors. The direct correlation between VEGF-positive cell number in tumor and DTCs in BM was observed $(r=0.542 ; P<0.025)$. 
The tendency was only assessed for the correlation between CK-positive BM and number of CD-68-positive cells as well as MVD in primary tumor $(P>0.05)$.

\subsection{Activity of Gelatinases in Primary Tumor and Bone} Marrow and CK-Positive Cells in Bone Marrow. It was shown that the association between activity of MMP-2 in tumor and presence of DTCs in BM, in particular MMP-2 activity was $9.2 \pm 5.1 \mu \mathrm{g} / \mathrm{g}$ tissue in patients with DTCs in BM whereas MMP-2 activity, was $4.1 \pm 2.8 \mu \mathrm{g} / \mathrm{g}$ in patients without DTCs in BM $(P<0.05)$. The association of tumor MMP-9 activity with DTCs in BM was not found. At the same time activity of both gelatinases in BM was linked with DTCs in BM; in particular activities of MMP-2 and MMP-9 were 8.6 \pm 4.0 and $7.5 \pm 3.4 \mu \mathrm{g} / \mathrm{g}$ in patients with DTCs in BM and $2.8 \pm 1.4$ and $2.6 \pm 1.85 \mu \mathrm{g} / \mathrm{g}$ in patients without DTCs in BM $(P<0.05$ and $P<0.05$, resp.). It has to be noted that patients with category $\mathrm{M}_{0}$ were analyzed only.

3.5. Overall Survival of Patients with and without Tumor Cells in Bone Marrow and Treated and Not Treated with Adjuvant Chemotherapy. Overall survival (OS) of patients with category $\mathrm{M}_{0}$ with DTCs in BM was shorter than that of patients with category $\mathrm{M}_{0}$ and without DTCs in BM $(P=0.0497)$ (Figure 1$)$. It has to be noted that patients in both groups were operated only. It can indicate that the detection of DTCs in BM may be considered as obligatory procedure before the decision concerning further treatment, in particular of patients with category $\mathrm{M}_{0}$.

Median follow-up time was 19.4 (range, 2.6-60.85) months from diagnosis for all patients (mean $21.9 \pm 1.9$ ). OS was significantly shorter in patients with $\mathrm{M}_{0}$ category and with DTCs, compared with patients with no DTCs (log-rank test: $P<0.05)$. If overall survival was analyzed for all patients $\left(\mathrm{M}_{0}\right.$ and $\mathrm{M}_{1}$ categories) the difference was not statistically significant $(P>0.05)$.

Overall, 30 patients $(33.7 \%)$ died during follow-up. In 26 patients $(86.7 \%)$ death was related to gastric cancer. Of these, 20 patients $(66.7 \%)$ had DTCs in BM. Of 20 patients with DTCs in BM, 8 patients (40\%) had $\mathrm{M}_{0}$ category. Of 26 patients, who died, 13 (50\%) have received any kind of adjuvant chemotherapy, and 10 (38.5\%) had DTCs in BM (4 patients (40\%) had $\mathrm{M}_{0}$ category). Survival in these patients was significantly shorter in patients with $\mathrm{M}_{0}$ category with DTCs compared with those patients with no DTC: 22.5 versus 39.5 months (Student's test, $P<0.05$ ). Survival in all patients (with $\mathrm{M}_{0}$ and $\mathrm{M}_{1}$ categories) was almost the same (22.2 and 24.9 months, resp.) independently from DTCs in BM.

It was also found that OS of patients with DTCs in BM and Flt-1-positive cells in BM was significantly shorter than that of patients with no DTCs in BM, but with Flt-1-positive cells in BM $(P=0.0437)$ (Figure 2). Patients in both groups were categorized as $M_{0}$ and $M_{1}$ and treated with adjuvant chemotherapy. OS of patients with category $M_{0}$ was not influenced by Flt-1 positivity of BM $(P>0.05)$.

Moreover, it was evaluated that OS of patients with DTCs in BM and VEGF-positive tumor was significantly shorter than that of patients without DTCs in BM and VEGF-positive

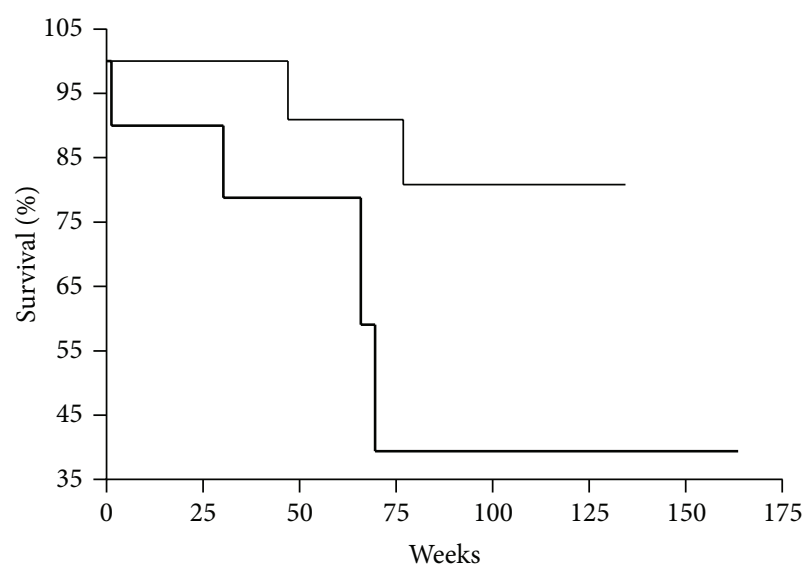

FIGURE 1: Kaplan-Meier overall survival curves for gastric cancer patients as a function of DTCs presence in bone marrow (DTCs ${ }^{-}$, thin line; DTCs ${ }^{+}$, bold line; $P<0.0497$ ). Patients with $\mathrm{M}_{0}$ category were analyzed and operated only.

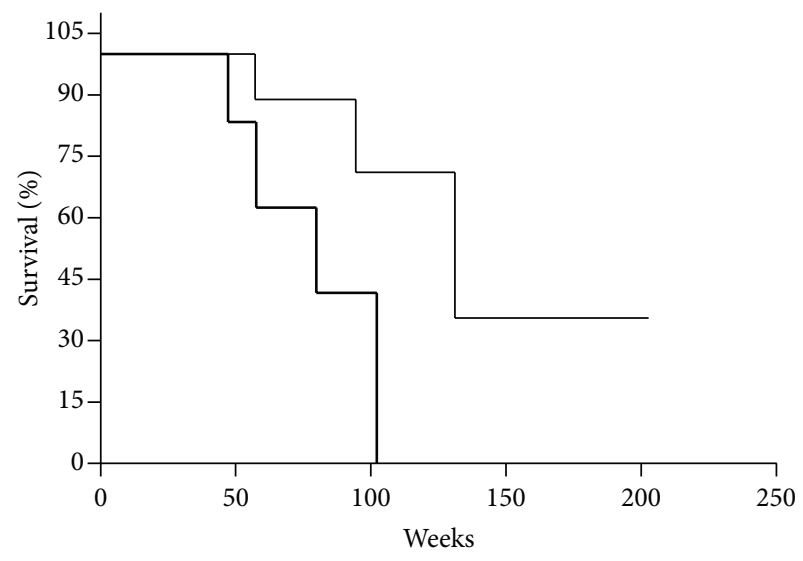

FIGURE 2: Kaplan-Meier overall survival curves for gastric cancer patients as a function of DTCs presence and Flt-1 expression in bone marrow $\left(\mathrm{DTCs}^{-} / \mathrm{Flt}^{-} 1^{+}\right.$, thin line; $\mathrm{DTCs}^{+} /$Flt $-1^{+}$, bold line; $P=$ 0.0437). Patients with $M_{0}$ and $M_{1}$ categories were analyzed and treated with operation and adjuvant chemotherapy.

tumor $(P=0.0486)$ (Figure 3$)$. The patients in both groups were treated with adjuvant chemotherapy and diagnosed as categories $M_{0}$ and $M_{1}$. It is relevant that OS of patients with category $\mathrm{M}_{0}$ and DTCs in BM and VEGF-positive tumor was significantly shorter than that of patients with category $M_{0}$ and without DTCs in BM and VEGF-positive tumor $(P=$ 0.0248) (Figure 4). Patients in both groups were treated with adjuvant chemotherapy.

It was also found that in patients with category $\mathrm{M}_{0}$ but with DTCs in BM who have been operated only risk of unfavorable outcome increased by a factor of $2(\mathrm{HR}=2.0$; 95\% CI $=0.98-5.76 ; P<0.05)$. These data can indicate the necessity of the additional diagnostic procedures for patients with $\mathrm{M}_{0}$ established by conventional methods because some of them may have DTCs in BM and need of adjuvant therapy. 


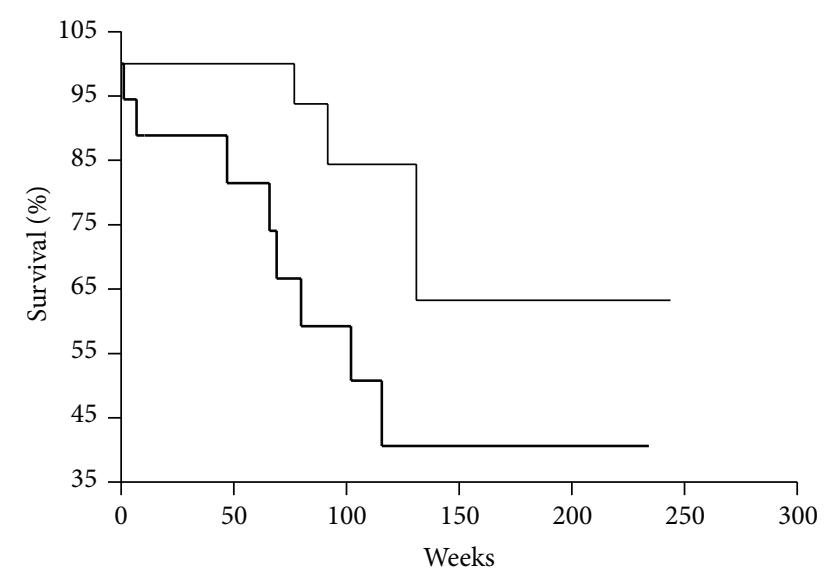

FIGURE 3: Kaplan-Meier overall survival curves for gastric cancer patients as a function of DTCs presence in bone marrow and VEGF expression in tumor $\left(\mathrm{DTCs}^{-} / \mathrm{VEGF}^{+}\right.$, thin line; $\mathrm{DTCs}^{+} / \mathrm{VEGF}^{+}$, bold line; $P<0.0486$ ). Patients with $\mathrm{M}_{0}$ and $\mathrm{M}_{1}$ categories were analyzed and treated with operation and adjuvant chemotherapy.

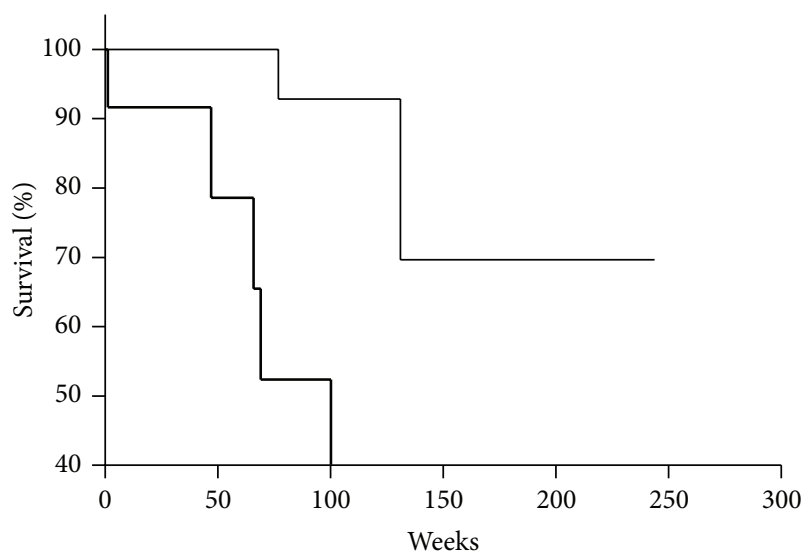

Figure 4: Kaplan-Meier overall survival curves for gastric cancer patients as a function of DTCs presence in bone marrow and VEGF expression in tumor $\left(\mathrm{DTCs}^{-} / \mathrm{VEGF}^{+}\right.$, thin line; $\mathrm{DTCs}^{+} / \mathrm{VEGF}^{+}$, bold line; $P=0.0248$ ). Patients with $\mathrm{M}_{0}$ category were analyzed and treated with operation and adjuvant chemotherapy.

\section{Discussion}

Our study found that tumor cells present in BM of 51.4\% patients with gastric cancer with category $\mathrm{M}_{0}$. DTCs in BM were detected in $35-60 \%$ of gastric cancer patients with category $\mathrm{M}_{0}$ by other authors [7]. It has to be noted that there are not enough publications concerning the significance of DTCs in BM in patients with $\mathrm{M}_{0}$. At the same time, namely, this information is more relevant than that in regard to patients with $M_{1}$ that are diagnosed by conventional methods. It should be noted that association between DTCs in $\mathrm{BM}$ and the level of hypoxia in the primary tumor was found: tumor cells in BM were found in $80 \%$ of cases where severe hypoxia was found in the primary tumor, whereas under moderate and mild hypoxia DTCs they were found only in $20 \%$ of cases $(P<0.05)$. It was also determined that a probability of DTCs appearance in BM is increased by a factor of 11 (odds ratio $11.8,95 \%$ CI $3.0587-45.60, \chi^{2}=$ $14.79, P<0.001$ ) when primary tumors are characterized by severe hypoxia. These data indicate a possible positive impact of hypoxia-associated signaling pathways on the escape of tumor cells from the primary tumor and their dissemination into the BM to form a premetastatic niche as suggested by Kaplan et al. [28].

Close correlation was observed between DTCs and VEGF expression in primary tumor confirmed by early observation [15], but correlation between DTCs and tumor MVD was not found in contrast to other authors [14, 29]. In regard to Flt-1 expression in BM it has to be noted that our study confirmed data obtained by Mimori et al. [30] that simultaneous presence of tumor cells and Flt-1-positive cells in $\mathrm{BM}$ is clinically relevant for metastasis.

The role of MMPs in tumor dissemination is well known, but MMPs activity in BM of gastric cancer patients was not determined till now. Our study has shown that activity of MMP-2 and MMP-9 in BM of patients with $\mathrm{M}_{0}$ but with DTCs was increased by a factor of 3.1 and 2.9 , respectively. It allows suggesting that gelatinases may play a significant role in the formation of premetastatic niche, in particular, in the reorganization of cellular microenvironment in BM.

In conclusion, it may be summarized that $51.4 \%$ of gastric cancer patients categorized as $\mathrm{M}_{0}$ have tumor cells in BM. Presence of DTCs is correlated with level of tumor hypoxia and accompanied with Flt-1 positivity of BM. The probability of Flt-1 positivity of BM was increased by a factor of 2.7 in patients with severe and moderate hypoxia in tumor. The significant correlation between CK-positivity of BM and VEGF expression as well as MMP-2 activity in tumor was shown. There is relevant observation that activity of both gelatinases in BM correlated with presence of DTCs in $B M$ of patients with $M_{0}$ only. OS of patients with $M_{0}$ with DTCs in BM was significantly shorter than that in patients without DTCs. It has to be noted that patients in both groups were operated only. It was also evaluated that OS of patients with DTCs and Flt-1 positivity of BM was significantly shorter than that of patients without DTCs but with Flt-1-positive BM (these patients were treated with adjuvant chemotherapy). Detection of DTCs in gastric cancer patients may be as relevant indicator for personalised cancer therapy, in particular in the choice of treatment tactic for GC patients, especially with category $\mathrm{M}_{0}$. Perhaps, DTCs in $\mathrm{BM}$ may be as an argument to use adjuvant chemotherapy, though some other factors have to be taking into account, for instance, the influence of hostile microenvironment on DTCs dormancy in BM.

\section{Conflict of Interests}

The authors report no conflict of interests. The authors alone are responsible for the content and writing of the paper.

\section{Acknowledgment}

This research was supported by the National Academy of Sciences of Ukraine (Grants no. 2.2.5.309 and no. 0102U003228). 


\section{References}

[1] V. Catalano, R. Labianca, G. D. Beretta, G. Gatta, F. de Braud, and E. van Cutsem, "Gastric cancer," Critical Reviews in Oncology/Hematology, vol. 71, no. 2, pp. 127-164, 2009.

[2] Z. P. Fedorenko, A. V. Gajsenko, L. O. Gulak, E. L. Goroh, A. Yu. Ryzhov, O. V. Sumkina et al., "Cancer in Ukraine, 2010-2011," in Bulletin National Cancer Register Ukraine, vol. 13, pp. 28-29, 2012.

[3] P. Kienle and M. Koch, "Minimal residual disease in gastrointestinal cancer," Seminars in Surgical Oncology, vol. 20, no. 4, pp. 282-293, 2001.

[4] K. Pantel, C. Alix-Panabières, and S. Riethdorf, "Cancer micrometastases," Nature Reviews Clinical Oncology, vol. 6, no. 6, pp. 339-351, 2009.

[5] H. Lin, M. Balic, S. Zheng, R. Datar, and R. J. Cote, "Disseminated and circulating tumor cells: role in effective cancer management," Critical Reviews in Oncology/Hematology, vol. 77, no. 1, pp. 1-11, 2011.

[6] Y. Kang and K. Pantel, "Tumor cell dissemination: emerging biological insights from animal models and cancer patients," Cancer Cell, vol. 23, no. 5, pp. 573-581, 2013.

[7] C. Alix-Panabières, S. Riethdorf, and K. Pantel, "Circulating tumor cells and bone marrow micrometastasis," Clinical Cancer Research, vol. 14, no. 16, pp. 5013-5021, 2008.

[8] D. P. Dearnaley, J. P. Sloane, and M. G. Ormerod, "Increased detection of mammary carcinoma cells in marrow smears using antisera to epithelial membrane antigen," British Journal of Cancer, vol. 44, no. 1, pp. 85-90, 1981.

[9] W. Janni, F. D. Vogl, G. Wiedswang et al., "Persistence of disseminated tumor cells in the bone marrow of breast cancer patients predicts increased risk for relapse-a European pooled analysis," Clinical Cancer Research, vol. 17, no. 9, pp. 2967-2976, 2011.

[10] G. Schlimok, I. Funke, K. Pantel et al., "Micrometastatic tumour cells in bone marrow of patients with gastric cancer: methodological aspects of detection and prognostic significance," European Journal of Cancer, vol. 27, no. 11, pp. 1461-1465, 1991.

[11] K.-W. Jauch, M. M. Heiss, U. Gruetzner et al., "Prognostic significance of bone marrow micrometastases in patients with gastric cancer," Journal of Clinical Oncology, vol. 14, no. 6, pp. 1810-1817, 1996.

[12] E. Soeth, I. Vogel, C. Röder et al., "Comparative analysis of bone marrow and venous blood isolates from gastrointestinal cancer patients for the detection of disseminated tumor cells using reverse transcription PCR," Cancer Research, vol. 57, no. 15, pp. 3106-3110, 1997.

[13] H. Allgayer, M. M. Heiss, R. Riesenberg, R. Babic, K. W. Jauch, and F. W. Schildberg, "Immunocytochemical phenotyping of disseminated tumor cells in bone marrow by uPA receptor and CK18: investigation of sensitivity and specificity of an immunogold/alkaline phosphatase double staining protocol," Journal of Histochemistry \& Cytochemistry, vol. 45, no. 2, pp. 203-212, 1997.

[14] Y. Maehara, S. Hasuda, T. Abe et al., “Tumor angiogenesis and micrometastasis in bone marrow of patients with early gastric cancer," Clinical Cancer Research, vol. 4, no. 9, pp. 2129-2134, 1998.

[15] Y. Kakeji, Y. Maehara, K. Shibahara et al., "Clinical significance of micrometastasis in bone marrow of patients with gastric cancer and its relation to angiogenesis," Gastric Cancer, vol. 2, no. 1, pp. 46-51, 1999.
[16] R. Macadam, A. Sarela, J. Wilson, K. MacLennan, and P. Guillou, "Bone marrow micrometastases predict early postoperative recurrence following surgical resection of oesophageal and gastric carcinoma," European Journal of Surgical Oncology, vol. 29, no. 5, pp. 450-454, 2003.

[17] S. Gretschel, C. Schick, U. Schneider, L. Estevez-Schwarz, A. Bembenek, and P. M. Schlag, "Prognostic value of cytokeratinpositive bone marrow cells of gastric cancer patients," Annals of Surgical Oncology, vol. 14, no. 2, pp. 373-380, 2007.

[18] P. Kolodziejczyk, A. Pituch-Noworolska, G. Drabik et al., "The effects of preoperative chemotherapy on isolated tumour cells in the blood and bone marrow of gastric cancer patients," British Journal of Cancer, vol. 97, no. 5, pp. 589-592, 2007.

[19] G. de Manzoni, G. Pelosi, F. Pavanel et al., "The presence of bone marrow cytokeratin-immunoreactive cells does not predict outcome in gastric cancer patients," British Journal of Cancer, vol. 86, no. 7, pp. 1047-1051, 2002.

[20] Y. Fujita, M. Terashima, Y. Hoshino et al., "Detection of cancer cells disseminated in bone marrow using real-time quantitative RT-PCR of CEA, CK19, and CK20 mRNA in patients with gastric cancer," Gastric Cancer, vol. 9, no. 4, pp. 308-314, 2006.

[21] E. Oki, Y. Kakeji, H. Baba et al., "Clinical significance of cytokeratin positive cells in bone marrow of gastric cancer patients," Journal of Cancer Research and Clinical Oncology, vol. 133, no. 12, pp. 995-1000, 2007.

[22] F. C. Bidard, F. R. Ferrand, F. Huguet et al., "Disseminated and circulating tumor cells in gastrointestinal oncology," Critical Reviews in Oncology/Hematology, vol. 82, no. 2, pp. 103-115, 2012.

[23] P. Vaupel and A. Mayer, "Hypoxia in cancer: significance and impact on clinical outcome," Cancer and Metastasis Reviews, vol. 26, no. 2, pp. 225-239, 2007.

[24] International Union Against Cancer, TNM Classification of Malignant Tumors, edited by L. H. Sobin and C. Wittekind, Wiley-Liss, New York, NY, USA, 6th edition, 2002.

[25] C. Fenoglio-Preiser, F. Carneiro, P. Correa et al., "Gastric carcinoma," in World Health Organization Classification of Tumors. Tumours of the Stomach, S. R. Hamilton and L. A. Aaltonen, Eds., vol. 3, chapter 3, pp. 39-52, IARC Press, Lyon, France, 2000.

[26] N. Weidner, J. P. Semple, W. R. Welch, and J. Folkman, "Tumor angiogenesis and metastasis-correlation in invasive breast carcinoma," The New England Journal of Medicine, vol. 324, no. 1, pp. 1-8, 1991.

[27] S. Osinsky, L. Bubnovskaya, I. Ganusevich et al., "Hypoxia, tumour-associated macrophages, microvessel density, VEGF and matrix metalloproteinases in human gastric cancer: interaction and impact on survival," Clinical and Translational Oncology, vol. 13, no. 2, pp. 133-138, 2011.

[28] R. N. Kaplan, B. Psaila, and D. Lyden, "Bone marrow cells in the "pre-metastatic niche": within bone and beyond," Cancer and Metastasis Reviews, vol. 25, no. 4, pp. 521-529, 2006.

[29] K. Matsunami, T. Nakamura, H. Oguma, Y. Kitamura, and K. Takasaki, "Detection of bone marrow micrometastasis in gastric cancer patients by immunomagnetic separation," Annals of Surgical Oncology, vol. 10, no. 2, pp. 171-175, 2003.

[30] K. Mimori, T. Fukagawa, Y. Kosaka et al., "Hematogenous metastasis in gastric cancer requires isolated tumor cells and expression of vascular endothelial growth factor receptor-1," Clinical Cancer Research, vol. 14, no. 9, pp. 2609-2616, 2008. 


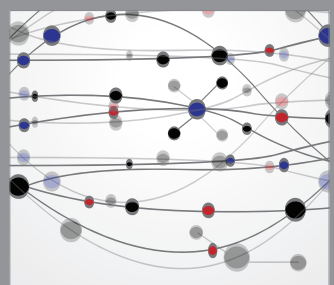

The Scientific World Journal
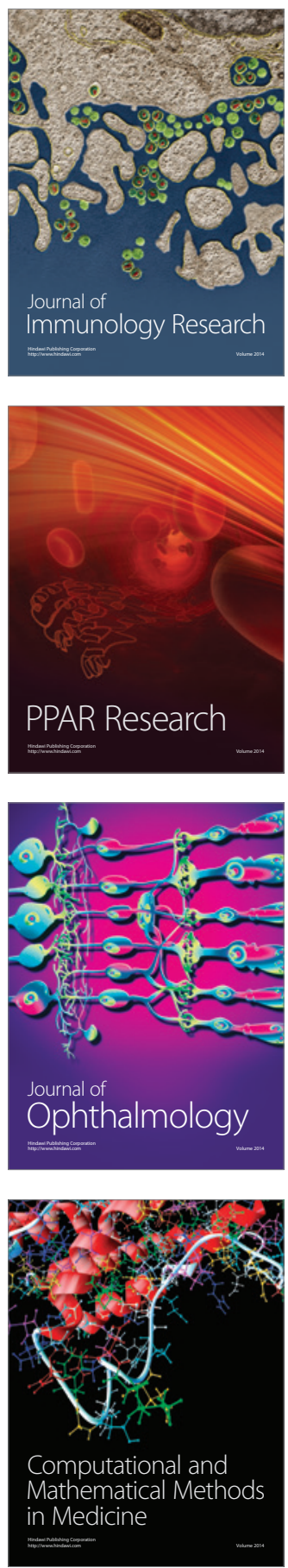

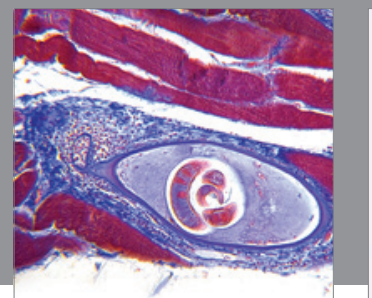

Gastroenterology

Research and Practice
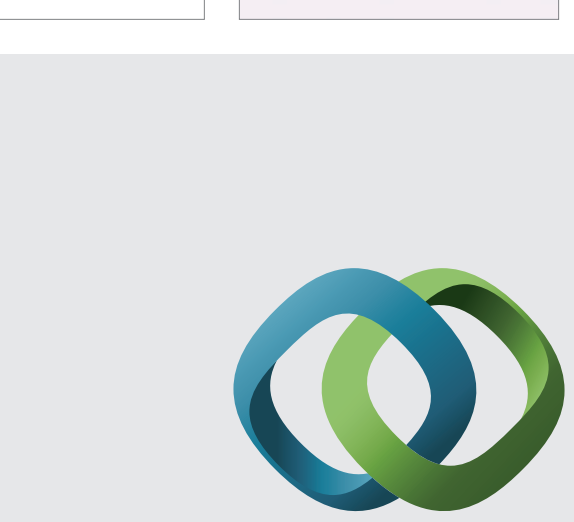

\section{Hindawi}

Submit your manuscripts at

http://www.hindawi.com
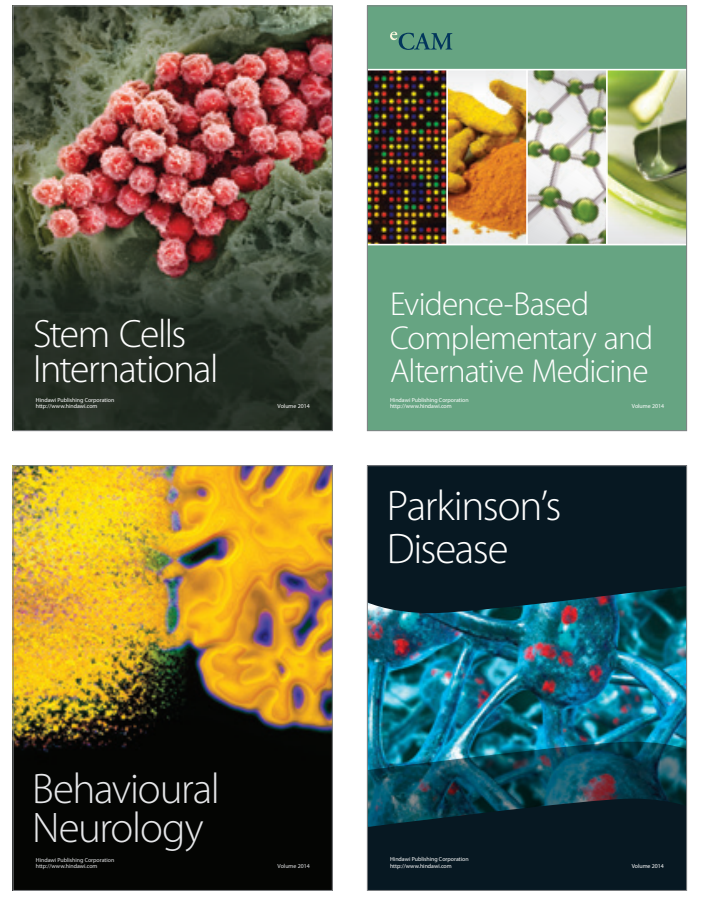
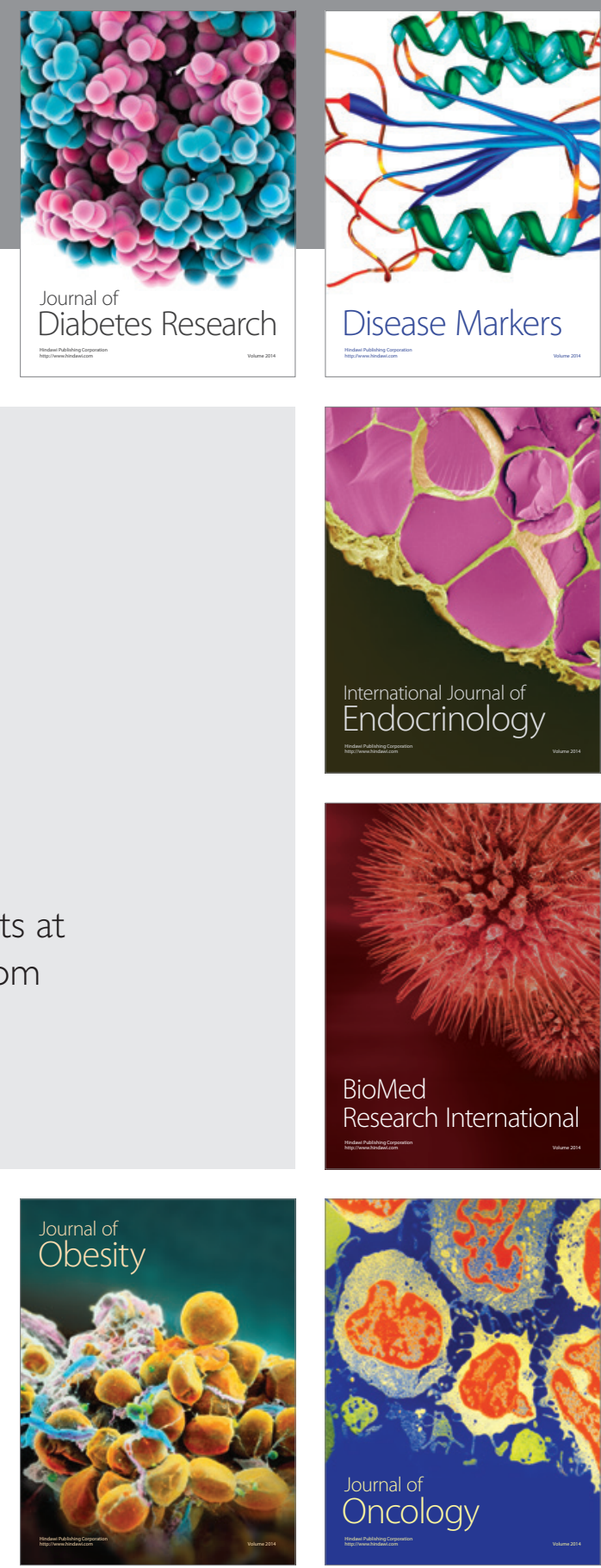

Disease Markers
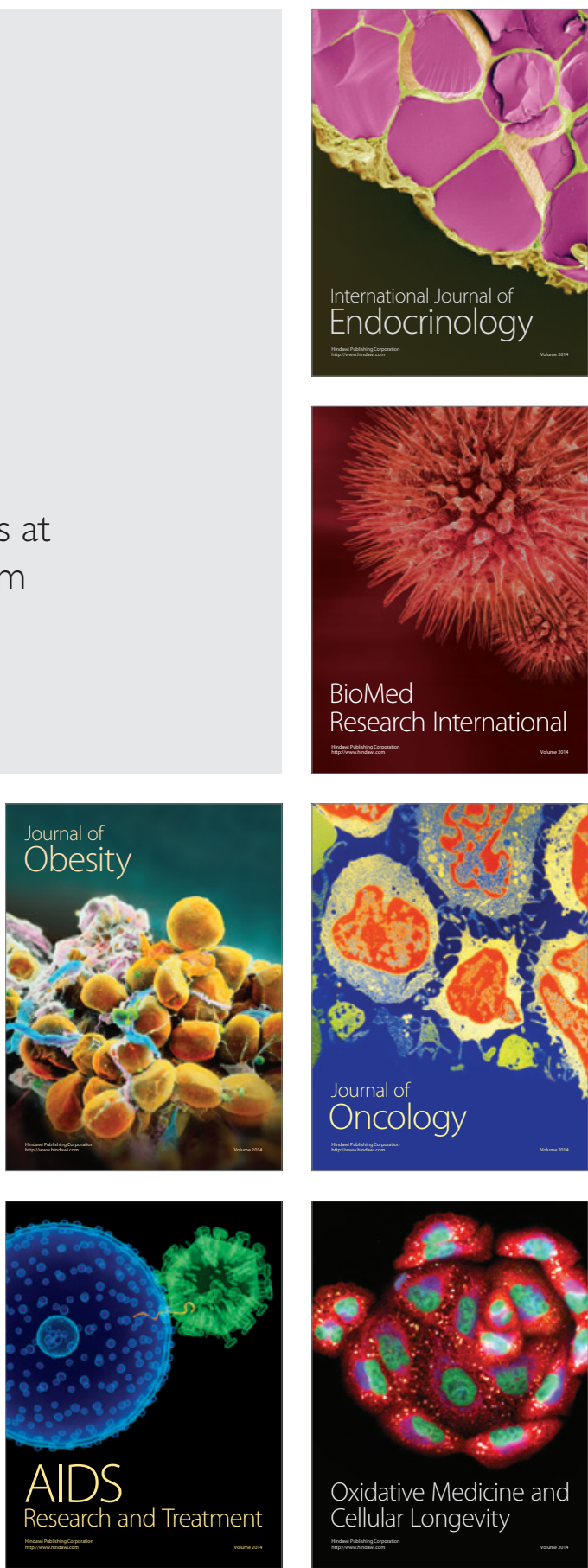